\title{
Evolution of the low-frequency spin dynamics in ferromagnetic manganites
}

\author{
J. A. Fernandez-Baca, ${ }^{1}$ P. Dai, ${ }^{1}$ H. Y. Hwang, ${ }^{2}$ S-W. Cheong, ${ }^{2,3}$ and C. Kloc ${ }^{2}$ \\ ${ }^{1}$ Oak Ridge National Laboratory, Oak Ridge, Tennessee 37831-6393 \\ 2 Bell Laboratories, Lucent Technologies, Murray Hill, NJ 07974 \\ ${ }^{3}$ Department of Physics and Astronomy, Rutgers University, Piscataway, New Jersey 08855
}

(September 23, 2018)

\begin{abstract}
Elastic and inelastic neutron scattering was used to study two ferromagnetic manganites $\mathrm{A}_{1-x} \mathrm{~B}_{x} \mathrm{MnO}_{3}(\mathrm{x} \approx 0.3)$ with $T_{c}=197.9 \mathrm{~K}$ and $300.9 \mathrm{~K}$. The spin dynamical behavior of these is similar at low temperatures, but drastically different at temperatures around $T_{c}$. While the formation of spin clusters of size $(\sim 20 \AA)$ dominates the spin dynamics of the $197.9 \mathrm{~K}$ sample close to $T_{c}$, the paramagnetic to ferromagnetic transition for the $300.9 \mathrm{~K}$ sample is more conventional. These results, combined with seemingly inconsistent earlier reports, reveal clear systematics in the spin dynamics of the manganites.
\end{abstract}

PACS numbers: 72.15.Gd, 75.30.Kz, 61.12.Gz 
The prospect of potential technological applications of the so-called colossal magnetoresistance (CMR) [1] has lead to a revival in the study of perovskite manganites $A_{1-x} B_{x} \mathrm{MnO}_{3}$ (where $A$ and $B$ are trivalent and divalent ion respectively). For compositions of $x \approx 0.3$, these materials exhibit a resistivity drop that is intimately related to the paramagnetic to ferromagnetic ordering at the Curie temperature $\left(T_{C}\right)$. The magnitude of the resistivity drop and $T_{C}$ can be tuned continuously by different $A$-site substitutions [2]. The central issue is whether the microscopic understanding of these systems is complete within the double exchange (DE) model [3] or requires additionally a strong Jahn-Teller based electronlattice coupling [4,5]. Experimentally, large oxygen lattice distortions have been found in $\mathrm{La}_{0.7} \mathrm{Ca}_{0.3} \mathrm{MnO}_{3}\left(T_{C} \sim 250 \mathrm{~K}\right)[6.8$, consistent with the existence of lattice and/or magnetic polarons ( $10-20 \AA$ ) [9, 10]. These results may also be related to the development of a prominent diffusive central peak close to $T_{C}$ in this compound [11]. By contrast, no signatures of unusual oxygen lattice distortions or unconventional spin dynamics have been reported [12 [14] in higher $T_{C}$ materials. In particular, Perring et al. [13] found that the spin wave dispersion throughout the Brillouin zone in a $\mathrm{La}_{0.7} \mathrm{~Pb}_{0.3} \mathrm{MnO}_{3}\left(T_{C}=355 \mathrm{~K}\right)$ crystal can be described by the conventional Heisenberg ferromagnet (FM) model with only the nearest-neighbor exchange coupling. Furukawa [15] has recently shown that this result can be fully explained within the DE model.

Thus, from the current experimental evidence it is difficult to develop a consistent picture of the spin dynamical behavior in the ferromagnetic perovskite manganites. Furthermore, it is not clear whether the reported polarons are the driving force of the CMR effect or merely a consequence of the insulator-to-metal transition from the DE mechanism itself.

By judicially tuning the lattice distortions through different $A$-site substitutions, we prepared two high quality perovskite manganite single crystals that have transition temperatures of $197.9 \mathrm{~K}\left(\mathrm{Nd}_{0.7} \mathrm{Sr}_{0.3} \mathrm{MnO}_{3}\right.$, or NSMO) and $300.9 \mathrm{~K}\left(\mathrm{Pr}_{0.63} \mathrm{Sr}_{0.37} \mathrm{MnO}_{3}\right.$, or PSMO). Our aim was to study, via elastic and inelastic neutron scattering, the systematics of the unique coupling between the lattice distortion and the spin dynamical properties of these systems. We show that the spin dynamical behavior of these two systems is similar at low temperature, but drastically different for temperatures around $T_{C}$. While the spontaneous formation of spin polarons $\left(\sim 20 \AA\right.$ in size) close to $T_{C}$ dominates the spin dynamics of the 197.7 K sample, the paramagnetic to ferromagnetic phase transition for the $300.9 \mathrm{~K}$ material is more conventional.

Large single crystals $(\approx 1 \mathrm{cc})$ of NSMO and PSMO of mosaic spreads of $\sim 0.5$ degree full-width at half-maximum (FWHM) were grown by the floating zone method. The neutron scattering experiments were carried out using the HB-1 triple-axis spectrometer at the High-Flux Isotope Reactor at the Oak Ridge National Laboratory. We have used pyrolytic graphite (PG) as monochromator and crystals of $\mathrm{PG}$ or Be as the analyzer to select neutrons with a wavelength of $2.46 \AA(E=13.6 \mathrm{meV})$ or $1.64 \AA(E=30.5 \mathrm{meV})$. The samples were slightly orthorhombic and twinned at the measured temperatures, but for the purpose of our measurements (and the utilized instrumental resolution) we assumed a cubic lattice $(a=3.86 \AA)$ for both crystals.

The temperature dependence of the intensity of the $\left(\begin{array}{lll}0 & 0 & 1\end{array}\right)$ Bragg reflection for both samples [see Figs 1(a) and 1(b)] indicates that ferromagnetic ordering develops at low temperatures. These measurements were done allowing sufficient time $(\approx 20 \mathrm{~min})$ for the samples to reach thermal equilibrium and were performed both on cooling (full circles) and on 
warming (open circles). There is a small but reproducible hysteresis in both data sets, suggesting that the ferromagnetic phase transition is weakly first order. The difference between the transition temperatures on cooling and on warming was less than $\approx 0.5 \mathrm{~K}$, and for the purposes of this experiment we chose the average of these two as the transition temperatures $(197.9 \pm 0.5 \mathrm{~K}$ for NSMO and $300.9 \pm 0.5 \mathrm{~K}$ for PSMO). The ferromagnetic-to-paramagnetic transition for NSMO is significantly rounder than that for PSMO, this may be due to the special features of the former system which will be addressed below. We also note that, within the precision of our measurements, there was no evidence of spin canting [16], or of a spin diffusive peak at the antiferromagnetic positions in any of the two samples.

The spin-wave dispersion curve for NSMO throughout the $(0,0,1+\zeta)$ Brillouin zone at $10 \mathrm{~K}$ is plotted in Figure 1(c) (solid circles), which also shows similar data for PSMO (open circles) [17. Note that despite the large difference in the transition temperatures of these two systems, the two curves are remarkably similar. This observation cannot be explained by any simple model of magnetic ordering. In particular, for a Heisenberg ferromagnet with only nearest neighbor exchange interaction $J, k T_{C}$ is nearly proportional to $J$ [18]. To elucidate the nature of the spin dynamics of these two systems, we performed high-resolution neutron inelastic scattering measurements. In the long wavelength limit (small wavectors q), the measured spin-wave energies can be fitted to the well-known quadratic dispersion relation $\hbar \omega=\Delta+D q^{2}$, where $D$ is the spin wave stiffness constant and $\Delta$ is a small dipolar gap. In the Heisenberg model, $D=2 J S a^{2}$, where $S$ is the magnitude of the electronic spin at the magnetic ion sites and $a$ is the lattice parameter. The quadratic dispersion form, however, is general and not limited to this model.

Figures 2(a) and 2(b) show the spin-wave energies vs. $q^{2}$ for PSMO and NSMO at various temperatures and the fits to the above quadratic dispersion relation. In all cases a very small $(\leq 0.05 \mathrm{meV})$ energy gap was obtained from the fits, but for practical purposes this gap was assumed to be zero. To follow the spin dynamical properties closer to the transition temperatures, we show in Figure 2(c) the spin-wave stiffness constant $D(T)$ as a function of $T / T_{C}$ for both samples. As $T \rightarrow T_{C}$, the hydrodynamic and the mode-mode coupling theories [19] predict a temperature dependence of the spin-wave stiffness of the form $D(T) \sim\left[\left(T-T_{C}\right) / T_{C}\right]^{\nu-\beta}$. The fit of the spin-wave data for PSMO to this form yields an exponent $(\nu-\beta) \approx 0.38 \pm 0.05$ [solid line in Fig. 2(c)]. This exponent should be compared to the value 0.34 predicted for a Heisenberg FM and to the measured values for iron $(0.36 \pm 0.03)$, cobalt $(0.39 \pm 0.05)$ or nickel $(0.39 \pm 0.04)$ [19]. The data for NSMO, on the other hand, show no evidence of the expected spin wave collapse at $\mathrm{T}_{c}$, just as in $\mathrm{La}_{0.67} \mathrm{Ca}_{0.33} \mathrm{MnO}_{3}$ [11]. Thus, while the low-temperature spin-wave stiffness for PSMO and NSMO is $D(0) \approx 165 \mathrm{meV}-\AA^{2}$ (very similar to the $170 \mathrm{meV}-\AA^{2}$ for $\mathrm{La}_{0.67} \mathrm{Ca}_{0.33} \mathrm{MnO}_{3}$ [11]), it is evident that the spin dynamics of these two systems becomes different as $T \rightarrow T_{C}$.

The striking differences between the spin-dynamical behavior for PSMO and NSMO as $T \rightarrow T_{C}$ are illustrated in Figure 3. The data shown in this figure are energy (constant-q) scans for an arbitrary wavevector $\mathrm{q}=0.08$ reciprocal lattice units (rlu). The peaks at the positive side of the energy axis correspond to neutron energy-loss while those at the negative side are for neutron energy-gain. A weak non-magnetic elastic incoherent contribution at $\hbar \omega=0$ has been subtracted. Both data sets show spin wave excitations of similar energies at the lower temperatures and these energies decrease as the temperature is increased. In PSMO [Fig. 3(a)], the excitations soften and become more intense as $T \rightarrow T_{C}$ as expected 
for a conventional FM. With increasing temperature, a central diffusive component develops. This central diffusive peak is possibly related to the longitudinal spin fluctuations from a Heisenberg FM at high temperatures, where the spin-wave description is not strictly valid [20], but it may already be a precursor of the spin dynamics seen in the lower $T_{C}$ NSMO, which is definitely unconventional. Above $\approx 0.91 T_{C}$, the spin wave energy renormalization in NSMO is slower than in PSMO, while the spin wave intensities damp dramatically and the excitation spectrum is dominated by the central $(\hbar \omega=0)$ component. This strong diffusive component, which persists at least up to $T=1.25 T_{C}$ with little $T$-dependence, has been attributed to the presence of magnetic polarons [9]. The magnetic nature of this diffusive component was verified by performing measurements close to the $\left(\begin{array}{lll}0 & 0 & 2\end{array}\right)$ Brillouin zone center which showed the expected intensity drop due to the Mn magnetic form factor.

To characterize the nature of the diffusive component of both samples, we performed systematic measurements of the static wavevector-dependent susceptibility $\chi_{\mathbf{q}}(\hbar \omega=0)$, and the static spin-correlation function. These were "elastic" neutron scattering measurements performed with and without energy analysis [19,20]. The temperature dependence of $\chi_{\mathbf{q}}(\hbar \omega=0)$ is shown in Figs. 4(a) and 4(b) for several selected wave vectors. For the PSMO, the susceptibility shows a sharp peak at the FM transition as expected for a conventional FM while the data for NSMO exhibits a broad peak with a maximum at a temperature somewhat below $T_{C}$. The profiles of the measured static spin-correlation function were least-squared fitted to an Ornstein-Zernike cross section [19] convoluted with the instrumental resolution. From these fits we obtained $\kappa(T)=1 / \xi(T)$, where $\xi(T)$ is the spin-correlation length. Figures $4(\mathrm{c})$ and $4(\mathrm{~d})$ show the temperature dependence of the spin correlation length $\xi$, and the insets show the corresponding temperature dependence of $\kappa(T)$ for both systems. For PSMO, the spin-correlation length $\xi(T)$ increases on cooling and diverges $(\xi(T)>100 \AA)$ close to $T_{C}$, indicating the onset of long-range FM order. In contrast, the spin-correlation length $\xi$ for NSMO is relatively insensitive to $T$ and remains small $(\approx 20 \AA)$ at $T_{C}$. $\xi(T)$ grows to over $100 \AA$ only at $T \approx 0.95 T_{C}$, consistent with the presence of magnetic polarons [9].

Our results clearly demonstrate that while the magnetism of these two ferromagnetic manganites in the low temperature metallic state may be similar, their spin dynamical behavior around the transition temperature can be drastically different. Therefore, it becomes clear that magnetism alone cannot explain the exotic spin dynamical properties in these systems and that the increased electron-lattice coupling plays a dominant role. In Table I we emphasize this point by summarizing the results of our experiments as well as those for $\mathrm{La}_{0.7} \mathrm{Sr}_{0.3} \mathrm{MnO}_{3}$ [12], $\mathrm{La}_{0.7} \mathrm{~Pb}_{0.3} \mathrm{MnO}_{3}$ [13] and $\mathrm{La}_{0.7} \mathrm{Ca}_{0.3} \mathrm{MnO}_{3}$ [11]. The various manganites in this table have been organized in order of decreasing $T_{C}$. For the three highest $T_{C}$ systems, the values of $D(0) / k T_{C}$ are characteristic of typical localized ferromagnets. However, larger $D(0) / k T_{C}$ values, more characteristic of itinerant ferromagnets [21], are observed in the last two systems. This systematic trend occurs despite the fact that with decreasing $T_{C}$, the zero temperature insulating state is approached.

We are grateful to D. Belanger, J. W. Cable, J. F. Cooke, H. A. Mook, S. E. Nagler, and R.M. Moon for helpful discussions. We would also like to acknowledge the expert technical support provided to us by R. G. Maples, S. Moore and G. B. Taylor. Oak Ridge National Laboratory is managed by Lockheed Martin Energy Research Corp. for the U.S. Department of Energy under contract number DE-AC05-96OR22464. 


\section{REFERENCES}

[1] S. Jin et al., Science 264, 413 (1994).

[2] H. Y. Hwang, S-W. Cheong, P. G. Radaelli, M. Marezzio, and B. Batlogg, Phys. Rev. Lett. 75, 914 (1995).

[3] C. Zener, Phys. Rev. 82, 403 (1951); P.W. Anderson and H. Hasegawa, ibid. 100, 675 (1955); K. Kubo and N. Ohata, J. Phys. Soc. Jpn. 33, 21 (1972); N. Furukawa, ibid. 64, 2734 (1995); 64, 2754 (1995).

[4] A. J. Millis et al., Phys. Rev. Lett. 74, 5144 (1995); ibid, 77175 (1996).

[5] H. Röder, Jun Zang and A. R. Bishop, Phys. Rev. Lett. 76, 1356 (1996).

[6] P. Dai, J. Zhang, H. A. Mook, S.-H. Liou, P. A. Dowben, and E. W. Plummer, Phys. Rev. B54, R3694 (1996).

[7] P. G. Radaelli, M. Marezzio, H. Y. Hwang, S-W. Cheong, and B. Batlogg, Phys. Rev. B54, 8992 (1996).

[8] S. J. L. Billinge, R. G. DiFrancesco, G. H. Kwei, J. J. Neuneier, and J. D. Thompson, Phys. Rev. Lett. 77, 715 (1996).

[9] J. M. de Teresa, M. R. Ibarra, P. A. Algarabel, C. Ritter, C. Marquina, J. Blasco, J. Garcia, A. del Moral, and Z. Arnold, Nature 386, 257 (1997).

[10] R. W. Erwin, J. Lynn, J. A. Borchers, J. L. Peng and R. L. Greene, J. Appl. Physics 815487 (1997).

[11] J. W. Lynn, R. W. Erwin, J. A. Borchers, Q. Huang, and A. Santoro, Phys. Rev. Lett. 76, 4046 (1996).

[12] M. Martin, G. Shirane, Y. Endoh, K. Hirota, Y. Moritomo and Y. Tokura, Phys. Rev. B 53, R14285 (1996).

[13] T. G. Perring, G. Aeppli, S. M. Hayden, S. A. Carter, J. P. Remeika, and S-W. Cheong, Phys. Rev. Lett. 77, 711 (1996).

[14] Y. Endoh and K. Hirota, J. Phys. Soc. Jpn. 66, 2264 (1997).

[15] N. Furukawa, J. Phys. Soc. Jpn. 65, 1174 (1996).

[16] P.G. DeGennes, Phys. Rev. 118, 141 (1960).

[17] H. Y. Hwang et al., unpublished.

[18] G. S. Rushbrooke et al. in Phase Transitions and Critical Phenomena, edited by C. Domb and M. S. Green (Academic, New York 1974).

[19] M. Collins in Magnetic Critical Scattering, (Clarendon, Oxford 1989).

[20] S. Lovesey in Theory of thermal neutron scattering from condensed matter, vol. 2, (Clarendon, Oxford, 1984).

[21] H. A. Mook, Neutron scattering studies of magnetic excitations in itinerant magnets, in Spin Waves and Magnetic Excitations I, edited by A. S. Borovik-Romanov and S. K. Sinha (Elsevier, Amsterdan, 1988), p. 444. 


\section{FIGURES}

FIG. 1. Temperature dependence of the ( $\begin{aligned} & 0 \\ & 0\end{aligned}$ 1) Bragg reflection of (a) PSMO, and (b) NSMO on cooling (full circles) and warming (open circles). Spin-wave dispersion curves of (c) NSMO (solid circles) and PSMO (open circles)throughout the zone $(0,0,1+\zeta)$ at $T=10 \mathrm{~K}$. The data for PSMO are from Ref. [17].

FIG. 2. Spin-wave energies vs. $q^{2}$ for (a) PSMO, and (b) NSMO. (c) Temperature dependence of the spin-wave stiffness constant $D(T)$ vs. $T / T_{C}$ for PSMO (open circles) and NSMO (closed circles). The solid line is the fit to the mode-mode coupling and hydrodynamic theories at high temperatures. The dashed line is an extrapolation to $T=0$ from the low-temperature mode-mode coupling theory.

FIG. 3. Energy scans for (a) PSMO, and (b) NSMO at $q=0.08$ rlu using the same spectrometer setup.

FIG. 4. (a) Temperature dependence of the static wave vector-dependent susceptibility $\chi_{\mathbf{q}}(\hbar \omega=0)$ for PSMO at $\mathrm{q}=0.027,0.033$ and $0.039 \mathrm{rlu}$. (b) $\chi_{\mathbf{q}}(\hbar \omega=0)$ for NSMO at $\mathrm{q}=0.025,0.030$ and 0.035 rlu. Temperature dependence of the spin correlation length for (c) PSMO, and (d) NSMO. Insets: temperature dependence of the inverse spin-correlation length. 


\section{TABLES}

\begin{tabular}{clcc}
\hline \hline Manganite & $T_{C}(\mathrm{~K})$ & $D(0)\left(\mathrm{meV}-\AA^{2}\right)$ & $D(0) / k T_{C}\left(\AA^{2}\right)$ \\
\hline $\mathrm{La}_{0.7} \mathrm{Sr}_{0.3} \mathrm{MnO}_{3}$, Ref. [13] & 378 & 188 & 5.8 \\
$\mathrm{La}_{0.7} \mathrm{~Pb}_{0.3} \mathrm{MnO}_{3}$, Ref. [14] & 355 & 134 & 4.4 \\
$\mathrm{Pr}_{0.63} \mathrm{Sr}_{0.37} \mathrm{MnO}_{3}$, this work & 300.9 & 165 & 6.4 \\
$\mathrm{La}_{0.7} \mathrm{Ca}_{0.3} \mathrm{MnO}_{3}$, Ref. [11] & 250 & 170 & 7.9 \\
$\mathrm{Nd}_{0.7} \mathrm{Sr}_{0.3} \mathrm{MnO}_{3}$, this work & 197.9 & 165 & 9.7 \\
\hline \hline
\end{tabular}

TABLE I. Summary of magnetic properties of various perovskite manganites $A_{1-x} B_{x} \mathrm{MnO}_{3}$ $(x \approx 0.3)$ in order of decreasing $T_{C}$. 

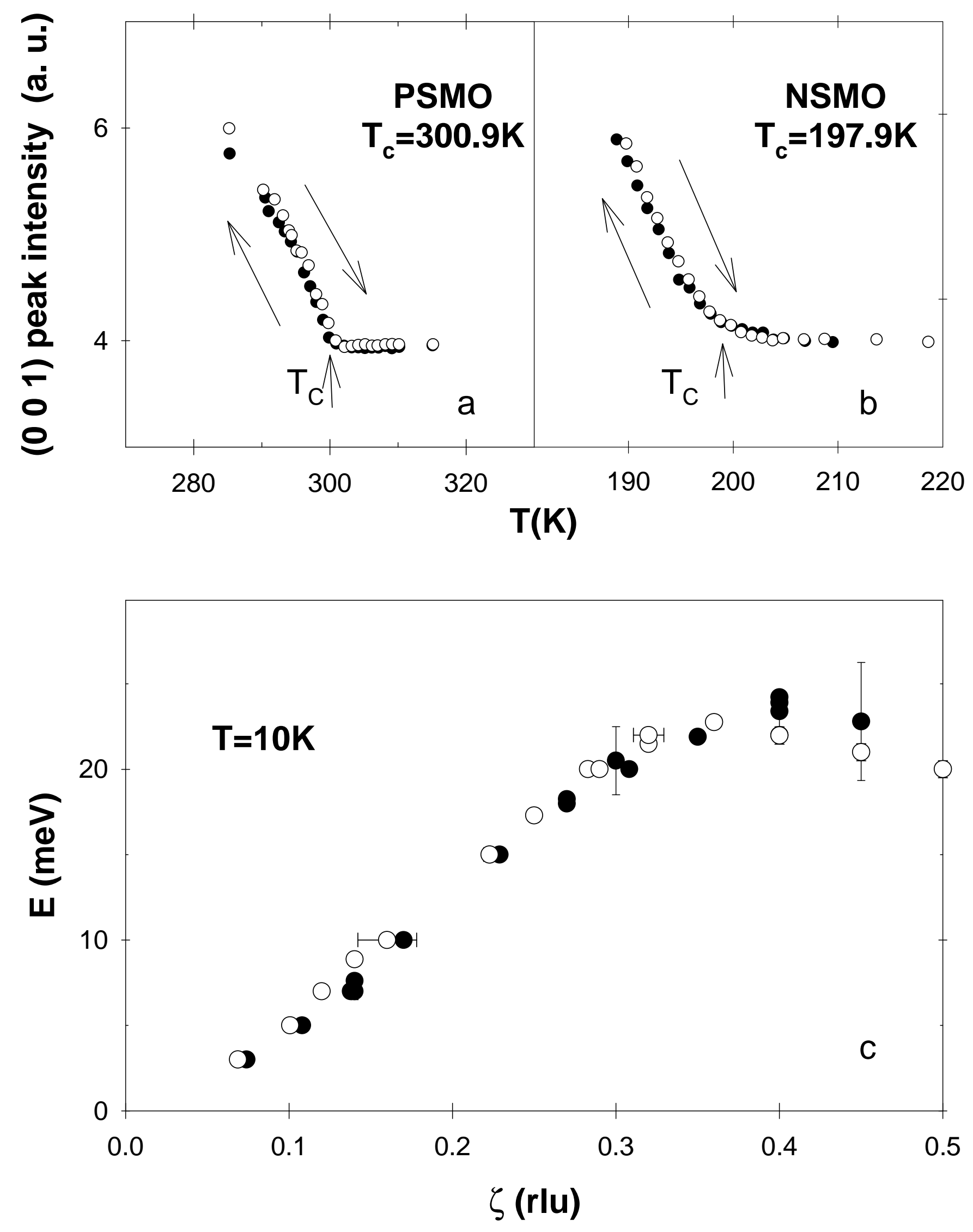

Figure 1. Fernandez-Baca et al 

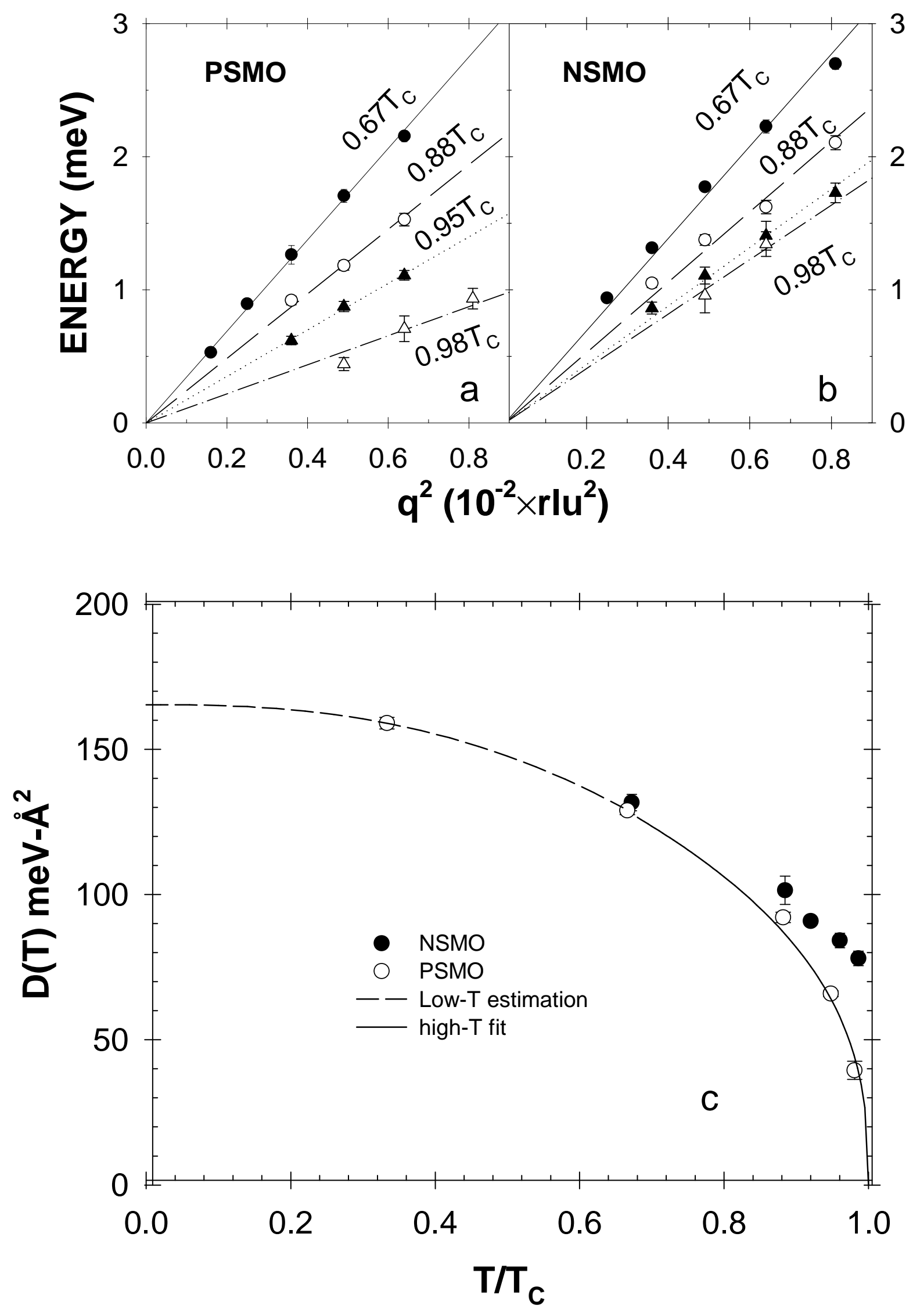

Figure 2. Fernandez-Baca et al. 


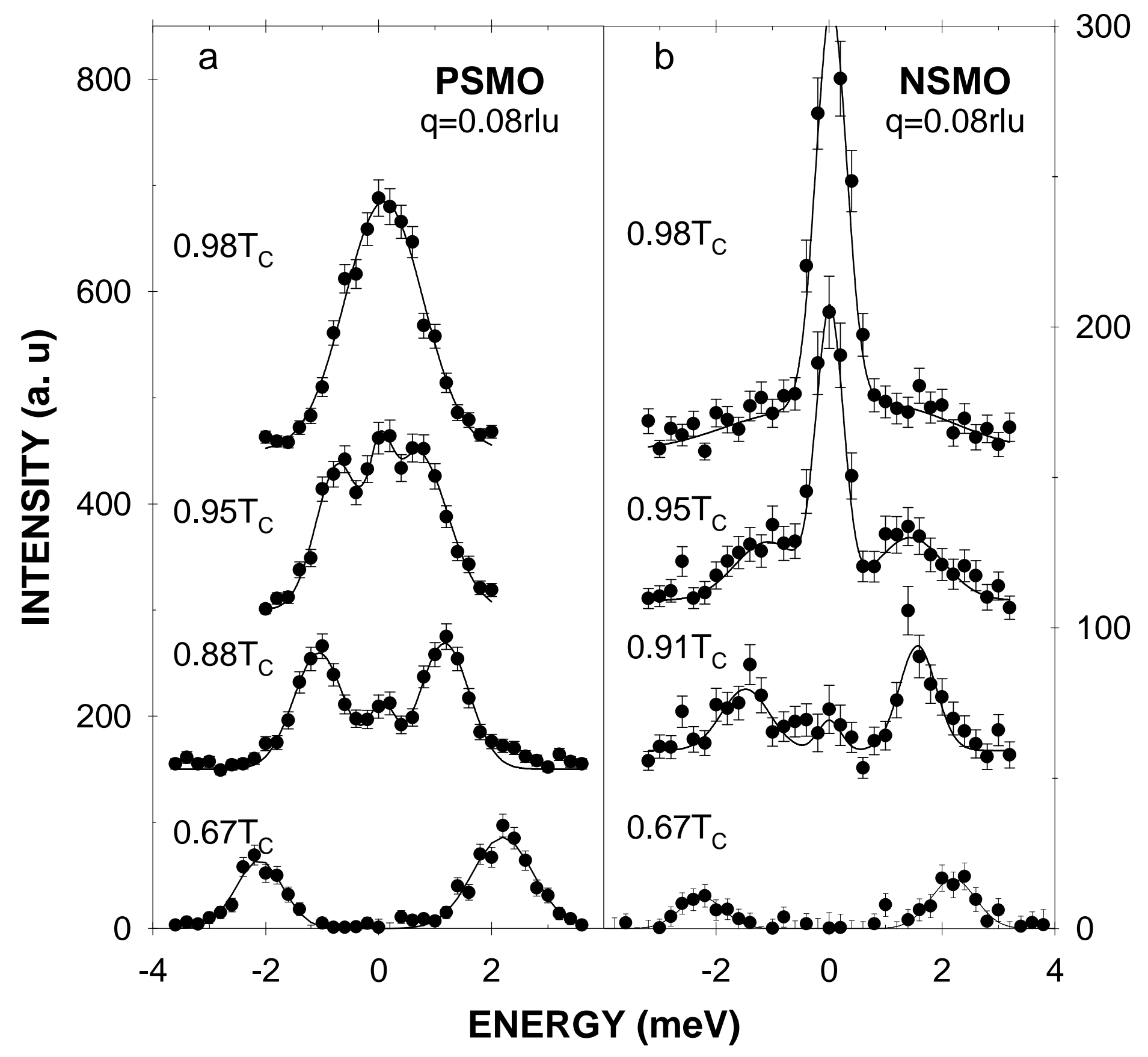

Figure 3. Fernandez-Baca et al 


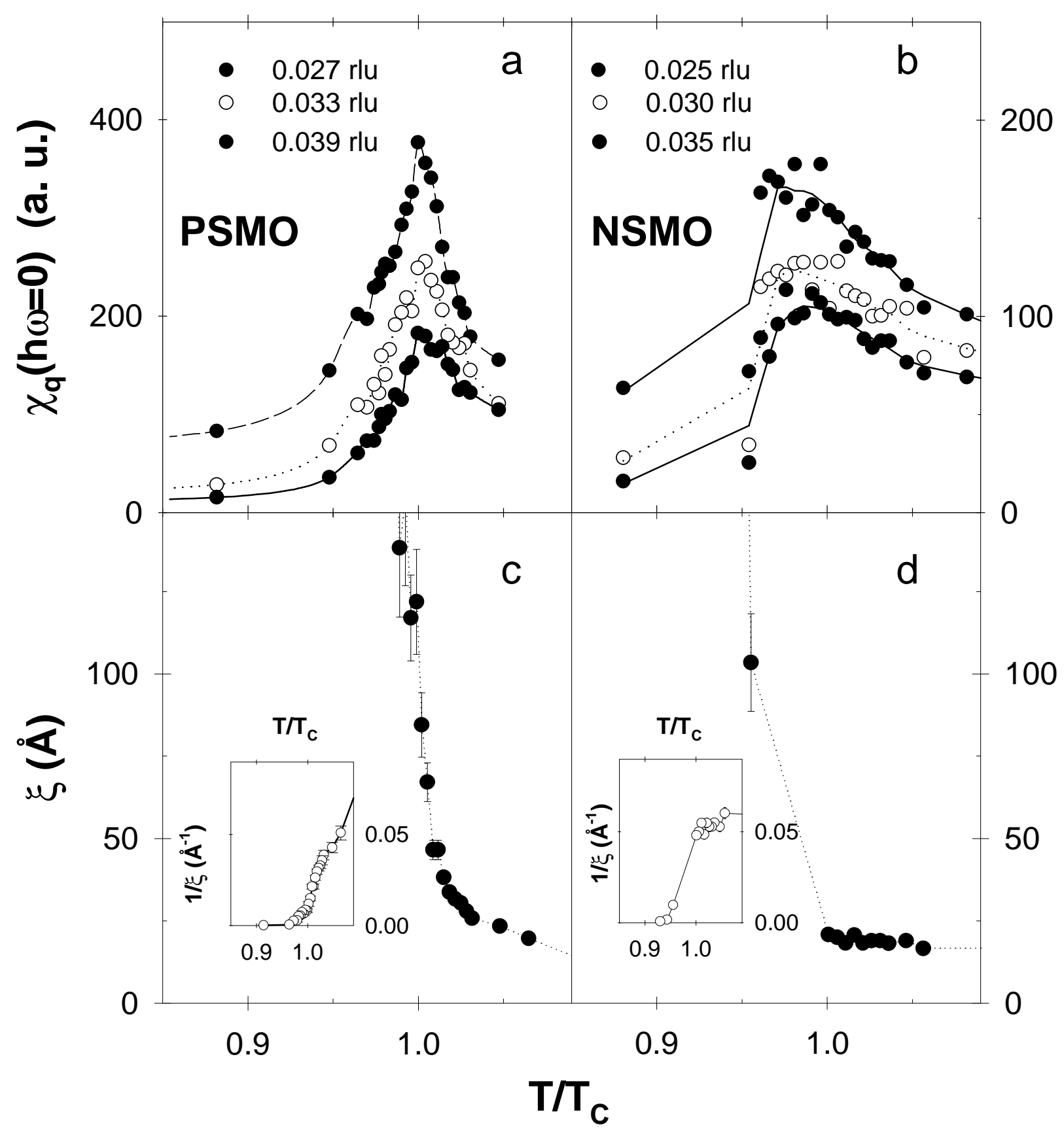

Figure 4. Fernandez-Baca et al 